D) Check for updates

Cite this: Food Funct., 2021, 12 10750

Received 8th July 2021, Accepted 5th August 2021 DOI: $10.1039 / \mathrm{d} 1 \mathrm{fo} 02167 \mathrm{~b}$ rsc.li/food-function

\section{Alleviation of cognitive impairment by gut microbiota lipopolysaccharide production- suppressing Lactobacillus plantarum and Bifidobacterium longum in mice $\uparrow$}

\author{
Dong-Yun Lee, Yoon-Jung Shin, Jeon-Kyung Kim, (D) Hyo-Min Jang, \\ Min-Kyung Joo and Dong-Hyun Kim (D) *
}

\begin{abstract}
Bacterial lipopolysaccharide (LPS) is a risk factor for the outbreak of Alzheimer's disease. Therefore, we isolated Lactobacillus plantarum NK151 and Bifidobacterium longum NK173 from a human fecal bacteria collection, which inhibited Escherichia coli LPS production, and examined their effects on the Escherichia coli K1- or LPS-induced cognitive impairment in mice. Oral gavage of NK151, NK173, or their $(4: 1)$ mixture (NKm) significantly alleviated Escherichia coli K1-induced cognitive impairment-like behaviors in the Y-maze and novel object recognition tasks. Their treatments decreased IL-1 $\beta$, IL-6, and TNF- $\alpha$ expression and $\mathrm{NF}-\mathrm{KB}^{+} / \mathrm{lba1} 1^{+}$and $\mathrm{LPS}^{+} / \mathrm{Iba1}^{+}$cell populations in the hippocampus, while the brainderived neurotrophic factor $(\mathrm{BDNF})^{+} /$neuronal nuclei $(\mathrm{NeuN})^{+}$cell population and BDNF to proBNDF expression increased. They suppressed LPS-induced cognition impairment-like behaviors and neuroinflammation marker levels in the hippocampus. Treatment with them reduced Escherichia coli K1- or LPS-induced LPS and apolipoprotein E levels in the blood and inflammatory marker levels in the colon. Furthermore, treatment with them modulated fecal Proteobacteria, Bacteroidetes, and Verrucomicrobia populations. Of these gut bacteria, Bacteroidaceae, Odoribacteraceae, Lactobacillaceae, Bifidobacteriaceae, Rikenellaceae, Helicobacteraceae, and Deferribacteraceae are correlated with cognitive function and blood and fecal LPS levels. These findings suggest that NK151 and NK173 may alleviate cognitive impairment with colitis by upregulating NF- $\mathrm{KB}$-mediated BDNF expression through the suppression of fecal and blood bacterial LPS levels.
\end{abstract}

\section{Introduction}

Neurobiota Research Center, College of Pharmacy, Kyung Hee University, 26, Kyungheedae-ro, Dongdaemun-gu, Seoul 02447, Korea.E-mail: dhkim@khu.ac.kr $\dagger$ Electronic supplementary information (ESI) available: Table S1. Effects of NK151 and NK172 on the gut microbiota composition at the phylum level in mice with Escherichia coli K1-induced cognitive impairment. Table S2. Effects of NK151 and NK172 on the gut microbiota composition at the family level in mice with Escherichia coli K1-induced cognitive impairment. Table S3. Effects of NK151 and NK172 on the gut microbiota composition at the genus level in mice with Escherichia coli K1-induced cognitive impairment. Table S4. Effects of NK151 and NK172 on the gut microbiota composition at the species level in mice with Escherichia coli K1-induced cognitive impairment. Table S5. Effects of NK151 and NK172 on the gut microbiota composition at the phylum level in mice with LPS-induced cognitive impairment. Table S6. Effects of NK151 and NK172 on the gut microbiota composition at the family level in mice with LPSinduced cognitive impairment. Table S7. Effects of NK151 and NK172 on the gut microbiota composition at the genus level in mice with LPS-induced cognitive impairment. Table S8. Effects of NK151 and NK172 on the gut microbiota composition at the species level in mice with LPS-induced cognitive impairment. Fig. S1. Effects of NK151 and NK173 on the fecal microbiota composition in mice with Escherichia coli K1-induced cognitive impairment. Fig. S2. Effects of NK151 and NK173 on the fecal microbiota composition in mice with LPSinduced cognitive impairment. See DOI: 10.1039/d1fo02167b
Dementia is a neurodegenerative disorder characterized by a progressive decline in behavioral and cognitive functions. ${ }^{1,2}$ The most common form of dementia in aged adults is Alzheimer's disease (AD). The pathogenesis of $\mathrm{AD}$ includes neuroinflammation, neurotransmitter imbalance, amyloidbeta $(A \beta)$ aggregation, and neurofibrillary tangle formation in the central nervous system. ${ }^{2-4} \mathrm{~A} \beta$ aggregation and neuroinflammation are promoted by gut dysbiosis and bacterial lipopolysaccharide (LPS) overproduction. ${ }^{5,6}$ Blood LPS levels in AD patients are significantly higher than in healthy control. ${ }^{7}$

The gut microbiota communicates with the brain through the microbiota-gut-brain (MGB) axis. ${ }^{6,8,9}$ Exposure to stressors such as antibiotics and immobilization causes gut dysbiosis and bacterial LPS overproduction. ${ }^{10,11}$ Excessive, chronic exposure to LPS induces inflammatory responses including NF- $\kappa \mathrm{B}$ activation and tumor necrosis factor (TNF)- $\alpha$ and interleukin (IL)-6 expression in the peripheral and systemic immune cells, inducing apolipoprotein E (apoE) in the blood 
and suppressing brain-derived neurotropic factor (BDNF) expression in the brain. ${ }^{12-14}$ Brain-derived neurotrophic factor (BDNF) regulates the neuronal synaptic plasticity in the peripheral and central nervous system. ${ }^{15,16}$ Moreover, blood BDNF is lower in $\mathrm{AD}$ patients than in healthy individuals. ${ }^{17,18}$ ApoE, a principal cholesterol carrier, directly binds LPS in the blood and delivers it to the liver, which degrades LPS. ${ }^{19,20}$ TNF- $\alpha$ expression suppressing Lactobacillus mucosae NK41 alleviates Escherichia coli K1-induced cognitive impairment in mice by

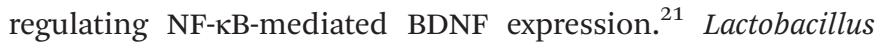
plantarum C29 improves cognitive impairment in 5XFAD and aged mice by modulating NF- $\kappa$ B-mediated BDNF expression. $^{22,23}$ Bifidobacterium longum NK46, which inhibits gut bacterial growth in vitro and NF- $\mathrm{BB}$ activation in BV-2 cells, suppresses cognitive decline in 5XFAD transgenic and aged mice by suppressing microbiota LPS-mediated NF- $\mathrm{B}$ activation. ${ }^{24}$ Lactobacillus brevis OW38 alleviates cognitive decline in aged mice by inhibiting gut microbiota LPS production and suppressing p16 expression. ${ }^{25}$ These findings suggest that the suppression of BDNF expression by LPS-mediated NF- $\kappa \mathrm{B}$ activation can cause cognitive impairment and reducing the overproduction of LPS by the gut microbiota and blood LPS level may be beneficial for the therapy of cognitive impairment.

The aim of the present study was to elucidate whether gut microbiota LPS production-suppressing probiotics could alleviate gut dysbiosis-induced cognitive impairment. Therefore, we isolated Lactobacillus plantarum NK151 and Bifidobacterium longum NK173, which inhibited the LPS production of Escherichia coli $\mathrm{K} 1$ in vitro, from the human fecal bacteria collection, and examined their effects on the Escherichia coli K1or LPS-induced cognitive impairment in mice.

\section{Materials and methods}

\subsection{Materials}

LPS (from Escherichia coli) and 4',6-diamidino-2-phenylindole dilactate (DAPI) were purchased from Sigma (St Louis, MO). The De Man, Rogosa, and Sharpe (MRS) medium was purchased from BD (Franklin Lakes, NJ). The general anaerobic medium (GAM) was purchased from Nissui Pharmaceutical Co. (Tokyo, Japan). The antibody for NF-кB was purchased from Cell Signaling Technology (Danvers, MA). Antibodies for CD11c, Iba1, and BDNF were purchased from Abcam (Cambridge, UK). The antibody for NeuN was purchased from Millipore (Burlington, MA). Alexa Fluor 488 and Alexa Fluor 488594 were purchased from Invitrogen (Carlsbad, CA). Enzyme-linked immunosorbent assay (ELISA) kits for IL-1 $\beta$, IL-10, and TNF- $\alpha$ were purchased from Ebioscience (Atlanta, GA). A Limulus Amebocyte Lysate (LAL) assay kit was purchased from Cape Cod Inc. (East Falmouth, MA). A QIAamp Fast DNA stool mini kit was purchased from Qiagen (Hilden, Germany). EasyTaq DNA polymerase and 100 bp plus II DNA ladder were purchased from TransGen Biotech (Beijing, China). TB Green ${ }^{\circledR}$ Premix Ex Taq ${ }^{\mathrm{TM}}$ II was purchased from Takara Bio (Shiga, Japan).

\subsection{Isolation of Escherichia coli K1 LPS production- suppressing probiotics}

Escherichia coli $\mathrm{K} 1$, which was isolated as a gut bacterium causing depression and memory impairment from human feces, ${ }^{21,26}$ and probiotics, which were selected from human fecal bacteria collection, were cultured in GAM broth at $37{ }^{\circ} \mathrm{C}$ for $24 \mathrm{~h}$. Thereafter, Escherichia coli $\mathrm{K} 1\left(1 \times 10^{5} \mathrm{CFU} \mathrm{mL}^{-1}\right)$ and probiotics $\left(1 \times 10^{4}\right.$ or $\left.1 \times 10^{6} \mathrm{CFU} \mathrm{mL}^{-1}\right)$ were simultaneously inoculated into GAM broth $(10 \mathrm{~mL})$ and anaerobically cultured at $37{ }^{\circ} \mathrm{C}$ for $24 \mathrm{~h}$. These cultured broths were centrifuged at $15000 \mathrm{~g}$ and at $4{ }^{\circ} \mathrm{C}$ for $20 \mathrm{~min}$. The resulting supernatant was successively filtered through $0.45 \mu \mathrm{m}$ and $0.22 \mu \mathrm{m}$ filters. The LPS level was measured in the supernatant using a LAL assay kit.

\subsection{Culture of Lactobacillus plantarum NK151 and Bifidobacterium longum NK173}

Gut bacteria including NK151 and NK173 were cultured in general media for probiotics, such as De Man, Rogosa and Sharpe (MRS) broth, and centrifuged at $5000 \mathrm{~g}$ and $4{ }^{\circ} \mathrm{C}$ for $20 \mathrm{~min}$, and washed with saline. For the in vivo experiment, the cells were collected, and freeze-dried and suspended in $1 \%$ maltose.

\subsection{Animals}

Specific pathogen-free C57BL/6 mice (male, 19-22 g, 6 weekold) were purchased from Koatech (Pyungtaek-Shi, Korea). All mice were housed in the plastic cage with the $5 \mathrm{~cm}$-raised wire floor under standard conditions (temperature, 20-22 ${ }^{\circ} \mathrm{C}$; humidity, $50 \pm 10 \%$ humidity; and light/dark cycle, $12 \mathrm{~h}$ ), fed standard laboratory chow and water ad libitum. Mice were acclimated for one week before being used in the experiment. Each group consisted of seven mice. All animal experiments were approved by The Committee for the Care and Use of Laboratory Animals in Kyung Hee University (IACUC No., KHUASP(SE)-20331) and performed according to The Kyung Hee University Guidelines for Laboratory Animals Care and Usage.

\subsection{Preparation of mice with cognitive impairment}

Mice with cognitive impairment were prepared, as previously reported. ${ }^{21,26}$ First, the mice were randomly divided into five groups (NC, EC, NK151, NK173, and NKm). Escherichia coli K1 (EC, $1 \times 10^{9} \mathrm{CFU}$ per mouse per day) were orally gavaged once a day for 5 days except for the NC group. In the NC group, 1\% maltose (vehicle) was orally gavaged instead of Escherichia coli K1. Test agents (NC, vehicle; EC, vehicle; NK151, $1 \times 10^{9} \mathrm{CFU}$ per mouse per day of Lactobacillus plantarum NK151; NK173, $1 \times 10^{9} \mathrm{CFU}$ per mouse per day of Bifidobacterium longum NK173; NKm, $1 \times 10^{9}$ CFU per mouse per day of NK151 and NK174 $[4: 1]$ mixture) were orally gavaged once a day for 5 days from the day after the final gavage of Escherichia coli K1. Second, mice were randomly divided into five groups (NC, LPS, NK151, NK173, and NKm). LPS (10 $\left.\mu \mathrm{g} \mathrm{kg}^{-1} \mathrm{day}^{-1}\right)$ was intraperitoneally injected once a day for 5 days except for the NC group. In the 
NC group, $1 \%$ maltose (vehicle) was intraperitoneally injected instead of LPS. Test agents (NC, vehicle; LPS, vehicle; NK151, 1 $\times 10^{9}$ CFU per mouse per day of Lactobacillus plantarum NK151; NK173, $1 \times 10^{9}$ CFU per mouse per day of Bifidobacterium longum NK173; NKm, $1 \times 10^{9}$ CFU per mouse per day of NK151 and NK173 [4:1] mixture) were orally gavaged once a day for 5 days from the day after the final injection of LPS.

For the assay of cognitive function, the Y-maze and novel object recognition (NOR) tasks were performed $20 \mathrm{~h}$ after the final gavage of test agents. The Y-maze was performed in a three-arm horizontal maze (40 $\mathrm{cm}$ long and $3 \mathrm{~cm}$ wide with $12 \mathrm{~cm}$-high walls) according to the method of Jeong et al. ${ }^{27} \mathrm{~A}$ mouse was initially placed within one arm and the sequence and number of arm entries were manually recorded for $8 \mathrm{~min}$. A spontaneous alternation was defined as entries into all three arms on consecutive choices. The ratio (\%) of actual to possible alternations was calculated. The NOR task was performed in the apparatus consisting of a dark-open field box $(45 \times 45 \times$ $50 \mathrm{~cm}$ ) according to the method of Jeong et $a .^{27}$ For the first trial, a mouse was placed in the box containing two identical objects and the frequency of touching each object was recorded for $10 \mathrm{~min}$. In the second trial conducted $24 \mathrm{~h}$ after the first trial, a mouse was placed in the box containing one of the old objects used in the first trial and a new object. Novel object recognition was calculated as the ratio of the number of times touching the new object to the sum of the touching frequencies.

Mice were sacrificed $3 \mathrm{~h}$ after the final behavioral task by $\mathrm{CO}_{2}$ inhalation. Blood, colon, brain, and stool were removed and stored at $-80^{\circ} \mathrm{C}$ for further experiments.

\subsection{Myeloperoxidase activity assay, ELISA, and immunoblotting}

Myeloperoxidase activity was assayed according to the method of Jang et al. ${ }^{11}$ The assay of corticosterone, apoE, IL-1 $\beta$, IL-10, and TNF- $\alpha$ expression levels were performed using commercial ELISA kits. ${ }^{21}$ p-p65, p65, BDNF, and $\beta$-actin expression levels were determined according to the method of Jang et al. ${ }^{11}$

\subsection{Immunofluorescence staining}

Immunofluorescence assay was performed, as reported previously. ${ }^{13,21}$ Mice were perfused transcardially with $4 \%$ paraformaldehyde. Their hippocampus and colon tissues were post-fixed with $4 \%$ paraformaldehyde, immersed in 30\% sucrose solution, frozen, and sectioned. The sections were incubated with antibodies for NF-אB $(1: 100)$, CD11c $(1: 200)$, Iba1 ( $1: 200)$, BDNF $(1: 200)$, and/or NeuN $(1: 200)$ overnight and then treated with Alexa Fluor 488 (1:200)- or Alexa Fluor 594 (1:200)-conjugated secondary antibody. The cell nucleus was stained with DAPI. The immunofluorescence-stained section was observed with a confocal microscope.

\subsection{Microbiota $16 S$ rRNA gene sequencing}

Bacterial genomic DNA was isolated from fresh mouse stool using a QIAamp DNA stool mini kit, as reported previously, ${ }^{21}$ and amplified using barcoded primers, which targeted the bacterial 16S rRNA V4 gene region. Amplicon sequencing was performed using Illumina iSeq 100 (San Diego, CA). Sequenced reads were stored in the NCBI's short read archive (accession number, PRJNA726149).

\subsection{Whole genome analysis}

The sequencing libraries were prepared according to the manufacturer's instructions of $20 \mathrm{~kb}$ Template Preparation using the BluePippin ${ }^{\mathrm{TM}}$ Size-Selection System using the PacBio DNA Template Prep Kit 1.0. NK151 genome sequence (5 contigs) was obtained by using the PacBio RSII platform and the complete NK173 genome sequence (1 contig) was obtained by using the PacBio Sequel platform.

\subsection{Statistical analysis}

Data values are indicated as the means \pm standard deviation (SD). The significance was analyzed by using one-way analysis of variance with Tukey's multiple comparison test $(p<0.05)$ and the correlation between the gut microbiota and cognitive function or the LPS level in the blood and feces was determined by Pearson's correlation coefficient, using Graph-Pad Prism 9 (GraphPad Software Inc., San Diego, CA).

\section{Results}

\subsection{NK151 and NK173 inhibited Escherichia coli K1 LPS production in vitro}

First, to select gut bacterial LPS production-inhibitory probiotics, we incubated Escherichia coli $\mathrm{K} 1$ with gut bacterial strains isolated from human feces. Of the tested gut bacteria, NK151 and NK173 suppressed the secretion of LPS from Escherichia coli K1 most strongly (Fig. 1). NK151 and NK173 were identified as Lactobacillus (Lactiplantibacillus) plantarum and
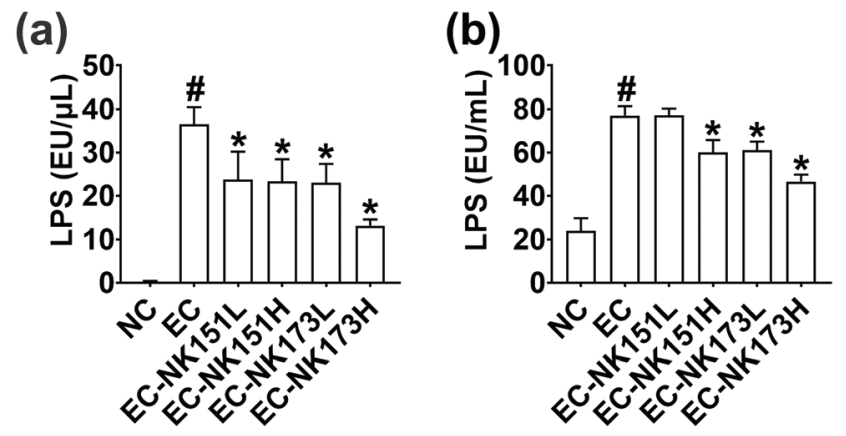

Fig. 1 Effects of NK151 and NK173 on the LPS production in Escherichia coli K1. (a) Effects on the LPS production in the intact bacteria (precipitate). (b) Effects on the LPS production in the supernatant. Escherichia coli K1 (EC, $1 \times 10^{5} \mathrm{CFU}$ ) in the absence or presence of NK151 or NK173 (l, $1 \times 10^{4} \mathrm{CFU}$; h, $1 \times 10^{6} \mathrm{CFU}$ ) was anaerobically cultured at $37^{\circ} \mathrm{C}$ for $24 \mathrm{~h}$ in general anaerobic medium. The LPS level was assayed in the supernatant of cultured media using an ELISA kit. Data represent the mean $\pm \mathrm{SD}$ ( $n=5$ per group). ${ }^{\#} p<0.05$ vs. NC; ${ }^{*} p<0.05$ vs. EC. 
Bifidobacterium longum, respectively, on the basis of the results of gram staining, API 50 CHL kit assay, 16S rDNA, and whole genome sequences. The genome of NK151 was 3288981 bp with a GC content of $44.5 \%$ (Fig. 2 and ESI Table S1 $\dagger$ ). The total number of CDS was 3930. The number of tRNA genes was 70 and of rRNA genes was 16. The genome sequence of NK151 showed phylogenetic similarity to Lactobacillus (Lactiplantibacillus) plantarum NCTC13644 (99.0\%), Lactobacillus (Lactiplantibacillus) argentoratensis DSM163565 (95.5\%), and Lactobacillus (Lactiplantibacillus) paraplantarum DSM10667 (86.1\%), using OrthoANI. The genome of NK173 was 2507434 bp with a GC content of 59.9\% (Fig. 2). The total number of CDS was 2077. The number of tRNA and rRNA genes was 59 and 12, respectively. The genome sequence of NK151 showed phylogenetic similarity to those of Bifidobacterium longum JCM1217 (98.6\%), Bifidobacterium longum DSM20211 (96.3\%), and Bifidobacterium longum ATCC15697 (95.0\%). NK151 and NK173 did not show hemolytic activity in the blood agar plate.

3.2. NK151 and NK173 alleviated cognitive function, colitis, and gut dysbiosis in mice with Escherichia coli K1-induced cognitive impairment

In the preliminary study, we examined the effects of NK151 at doses of $1 \times 10^{8}$ and $1 \times 10^{9}$ CFU per mouse per day against cognitive impairment in mice orally gavaged with Escherichia coli K1. NK151 at a dose of $1 \times 10^{9} \mathrm{CFU}$ per mouse per day significantly alleviated cognitive impairment in mice with Escherichia coli K1-induced cognitive impairment than at a dose of $1 \times 10^{8} \mathrm{CFU}$ per mouse, while it did not affect the cognitive function in control mice (data not shown). Therefore, we examined the effects of NK151 and NK173 at a dose of $1 \times 10^{9}$
(A)

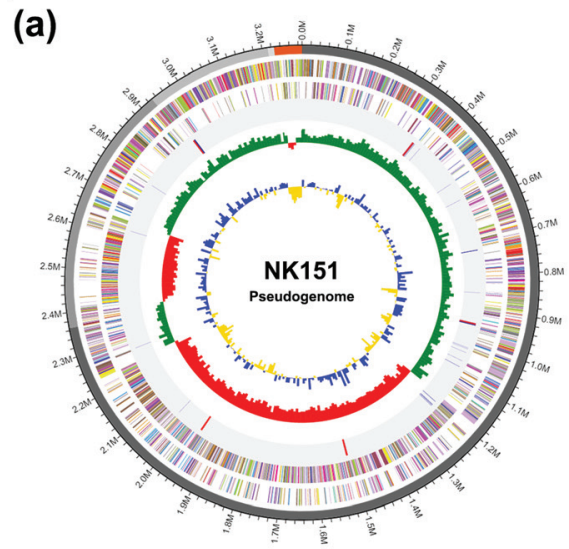

(b)

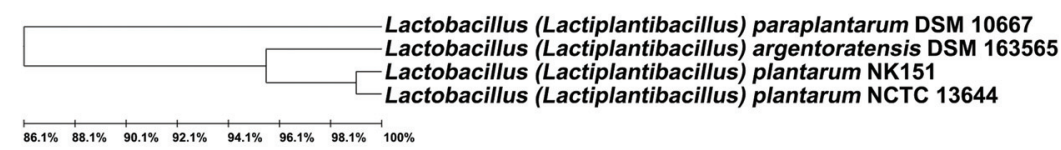

(c)

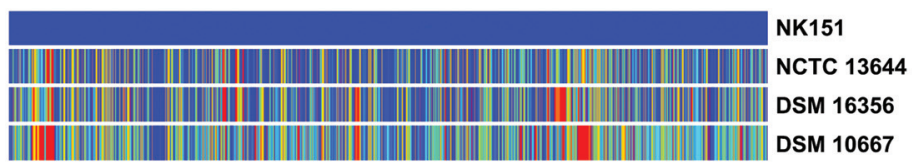

(B)

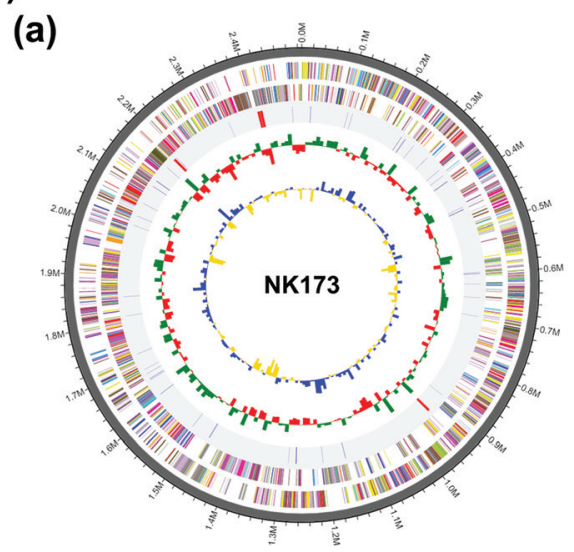

(b)

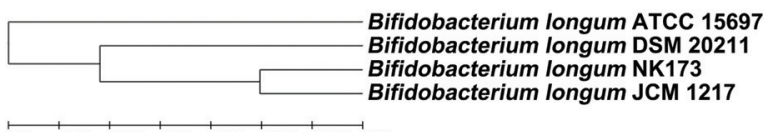

(c)

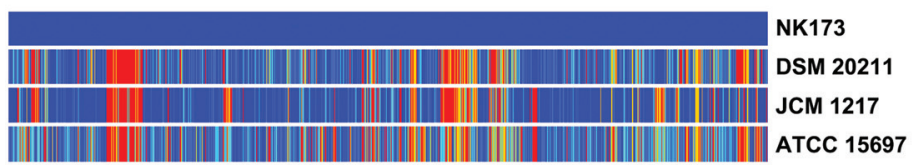

Fig. 2 Taxonomic classification by genome-wide comparative analysis of NK151 (A) and NK173 (B). (a) The pseudochromosome drawn from 5 contigs. The outermost circle indicates contig. The inner circle is the color coded for the CDS information analyzed in the forward strand, and the inner circle is the CDS information analyzed in the reverse strand. The fourth circle from the outside represents tRNA (blue) and rRNA (red). The inner circle indicates GC skew metric information (green, higher than the average; red, lower than the average). The innermost circle is GC ratio metric information (blue, higher values than average; yellow, lower values) GC skew and GC ratio metrics are displayed at $10 \mathrm{~kb}$ intervals. (b) Neighbor-joining tree based on the OrthoANI distance matrix (analyzed using UPGMA dendrogram, Newick format). (c) The pairwise ortholog matrix table (generated and colored according to the similarity between matching sequences). 
CFU per mouse per day on Escherichia coli K1-induced cognitive impairment in mice (Fig. 3). Oral gavage of Escherichia coli K1 significantly reduced the spontaneous alternation in the Y-maze task and exploration in the NOR task to $73.2 \%$ and $53.1 \%$ of the control group, respectively. However, oral administration of NK151, NK173, or NKm significantly alleviated Escherichia coli K1-suppressed spontaneous alternation to 93.4\%, 94.1\%, and $97.1 \%$ of the control group, respectively, and exploration to $77.4 \%, 102.4 \%$, and $109.7 \%$, respectively
(Fig. 3b and c). Furthermore, their treatments reduced Escherichia coli K1-induced IL-1 $\beta$ and TNF- $\alpha$ expression and NF- $\kappa \mathrm{B}$ activation in the hippocampus, while BDNF and IL-10 expression and BDNF to proBDNF expression ratio increased (Fig. 3d-g). They also reduced Escherichia coli K1-induced NF$\mathrm{\kappa B}^{+} / \mathrm{Iba}^{+}$and $\mathrm{LPS}^{+} / \mathrm{Iba}^{+}$cell populations, while the Escherichia coli $\mathrm{K} 1$-suppressed $\mathrm{BDNF}^{+} / \mathrm{NeuN}^{+}$cell population increased (Fig. 3h). Furthermore, they significantly decreased LPS and apoE levels in the blood (Fig. 3i and j). Oral gavage of (a)

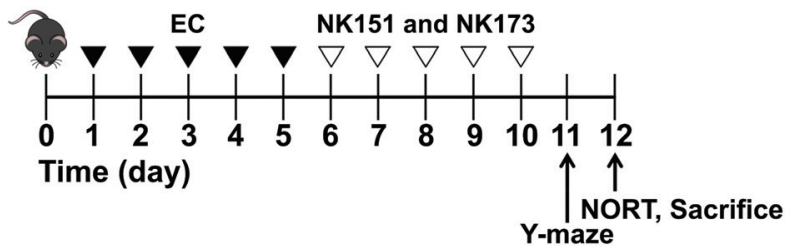

(b)

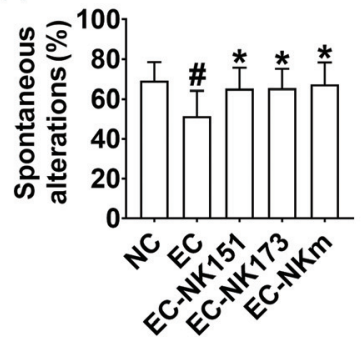

(c)

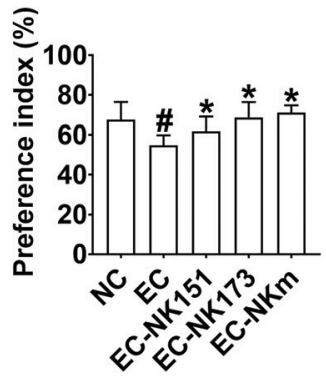

(d)

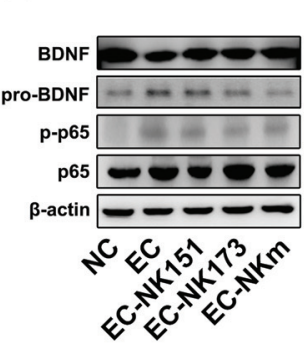

(h)

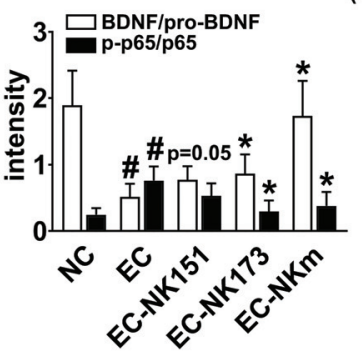

(e)

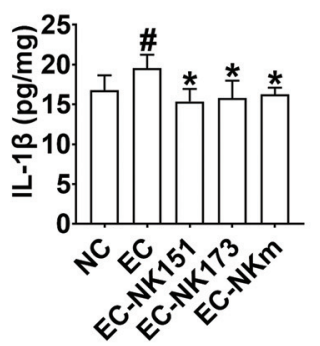

(f)

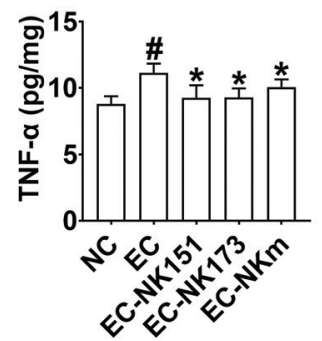

(g)

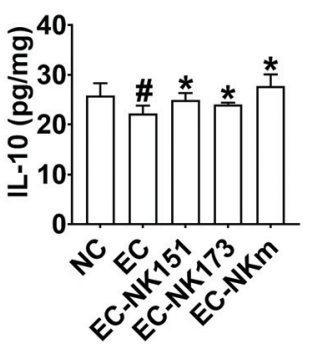

(i)

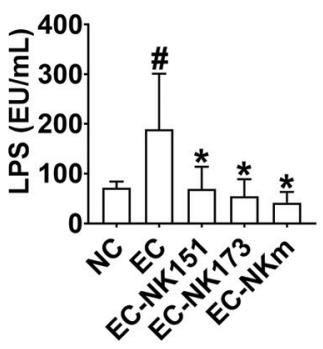

(j)

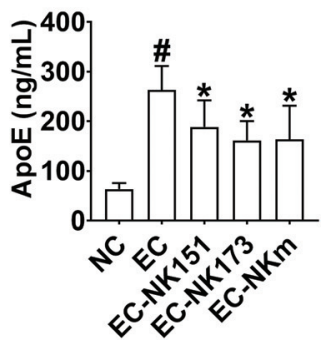

Fig. 3 NK151 and NK173 alleviated Escherichia coli K1-induced cognitive impairment in mice. (a) Experimental procedure. (b) Effects on the spontaneous alternation in the $\mathrm{Y}$-maze task. (c) Effects on the exploration (preference index) in the novel object recognition task. Effects on the BDNF and pro-BDNF expression, NF-KB activation, and their intensities (d), IL-1 $\beta$ (e), TNF- $\alpha$ (f), and IL-10 expression (g) and BDNF $/ \mathrm{NNeuN}^{+}, \mathrm{LPS}^{+} / \mathrm{Iba1}^{+}$, and $\mathrm{NF}-\mathrm{KB}^{+} / \mathrm{Iba1}^{+}$cell populations ( $\mathrm{h}$ ) in the hippocampus. Effects on the LPS (i) and APOE levels (j) in the blood. Cytokines were assayed using ELISA kits. NK151, NK173, their mixture (NKm), or Escherichia coli K1 (EC, $1 \times 10^{9} \mathrm{CFU}$ per mice per day) was orally gavaged. The control group (NC) was treated with the vehicle instead of test agents. Data values were described as mean \pm SD $(n=7) .{ }^{*} p<0.05$ vs. NC. ${ }^{*} p<0.05$ vs. EC. 
Escherichia coli K1 also caused colitis (Fig. 4). However, oral administration of NK151 and/or NK173 significantly alleviated the Escherichia coli K1-induced colon shortening, macroscopic score, myeloperoxidase activity, IL-1 $\beta$ and TNF- $\alpha$ expression, $\mathrm{NF}-\kappa \mathrm{B}$ activation, and NF- $\mathrm{BB}^{+} / \mathrm{Iba}^{+}$cell population, while IL-10 expression was increased. Furthermore, they decreased the Escherichia coli K1-induced LPS level in the feces. Of these, NKm alleviated Escherichia coli K1-induced neuroinflammation and colitis most potently.

Next, we investigated the effect of NKm on the fecal microbiota composition in mice with Escherichia coli K1-induced cognitive impairment. Oral gavage of Escherichia coli K1 shifted the $\alpha$-(OTUs) and $\beta$-diversities (PCOA) (Fig. 5A and ESI Tables S2-S5†). However, treatment with NK151 and/or NK173 partially restored the $\alpha$ - and $\beta$-diversities of the gut microbiota. At the phylum level, oral gavage of Escherichia coli K1 decreased Firmicutes and Verrucomicrobia populations, while the Bacteroidetes, Proteobacteria, and Cyanobacteria populations increased. Treatment with Escherichia coli $\mathrm{K} 1$ also increased Rikenellaceae and AC160630_f populations at the family level; Alistipes, Helicobacter, PAC001068_g and PAC000186_g populations at the genus level; and EF097112_s, and PAC001064_s populations at the species level, while it decreased the Lactobacillaceae, Lachnospiraceae, Akkermansiaceae, Bifidobacteriaceae, and Dehalobacterium_f populations at the family level; Lactobacillus, LLKB_g, and PAC001092_g populations at the genus level; and the Lactobacillus reuteri group, PAC001093_s, and AB626939_S populations at the species level. However, treatment by oral administration of NK151 and/or NK173 suppressed Escherichia coli K1-induced Proteobacteria at the phylum level; Rikenellaceae, Christensenellaceae, and AC160630_f populations at the family level, Alistipes and Helicobacter populations in the genus level; and PAC001071_s population at the species level and increased Escherichia coli K1-suppressed Verrucomicrobia and Firmicutes populations at the phylum level; Lactobacillaceae and Bifidobacteriaceae populations at the family level, Lactobacillus, LLKB_g, and PAC001092_g (a)

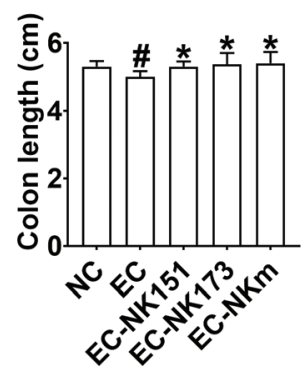

(d)

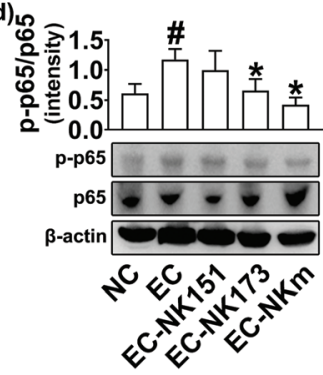

(g)

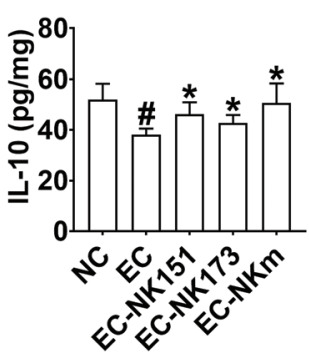

(b)

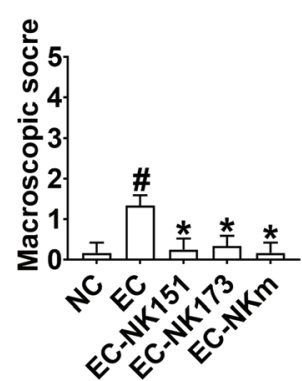

(e)

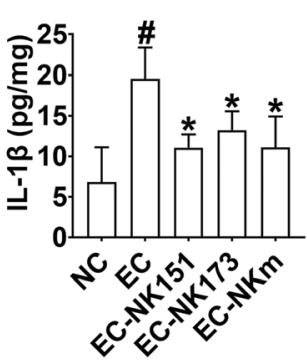

(h)

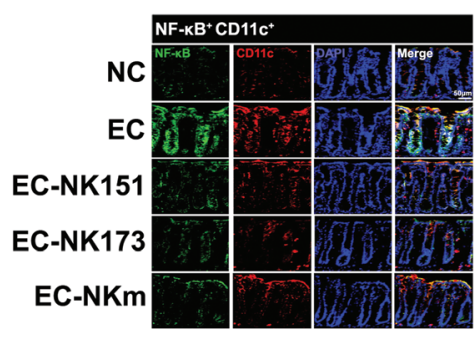

(c)

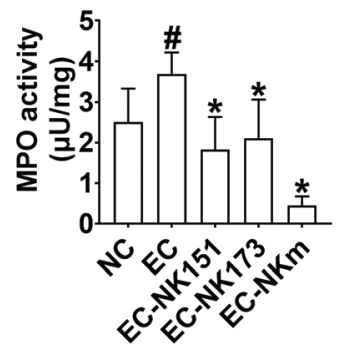

(f)

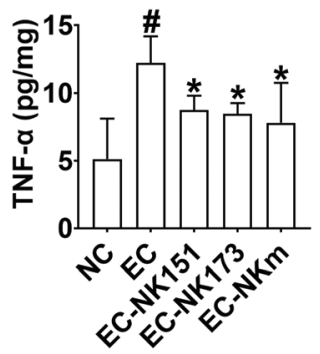

(i)

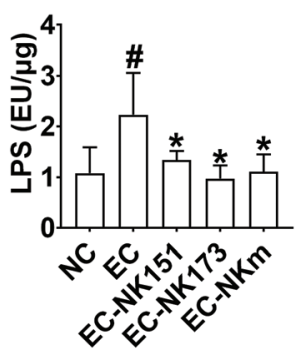

Fig. 4 NK151 and NK173 alleviated colitis in mice with Escherichia coli K1-induced cognitive impairment. (a) Effects on the colon length. (b) Effects on the macroscopic score. (c) Effects on the myeloperoxidase activity in the colon. Effects on the NF-KB activation (d), IL-1 $(e)$, TNF- $\alpha$ ( $f$ ), and IL-10 expression (g) and $\mathrm{NF}-\mathrm{KB}^{+} / \mathrm{CD} 11 \mathrm{c}^{+}$cell population (h) in the colon. (i) Effects on the LPS in the feces. Cytokines were assayed using ELISA kits. NK151, NK173, their mixture (NKm), or Escherichia coli K1 (EC, $1 \times 10^{9} \mathrm{CFU}$ per mice per day) was orally gavaged. The control group (NC) was treated with the vehicle instead of test agents. Data values were described as mean \pm SD $(n=7) .{ }^{\#} p<0.05$ vs. NC. ${ }^{*} p<0.05$ vs. EC. 
(A)

(a)

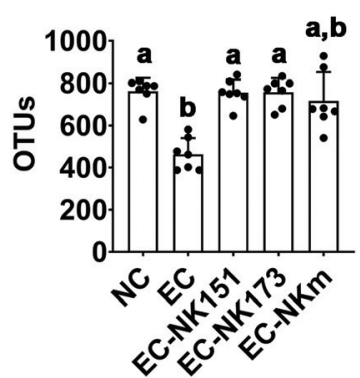

(b)

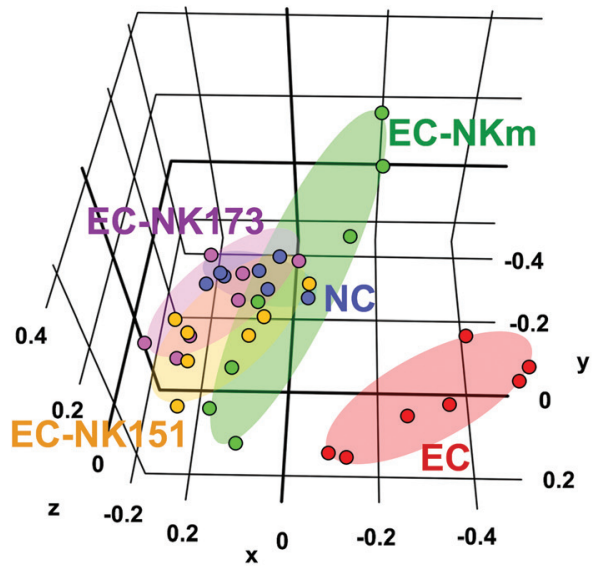

(c)

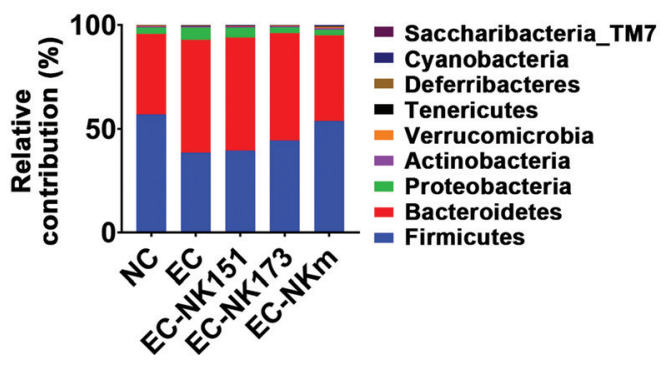

(C)

(a)

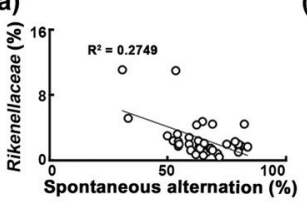

(b)

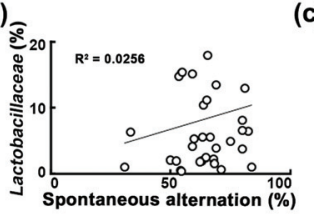

(c)

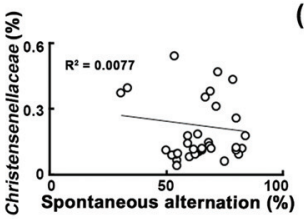

(d)

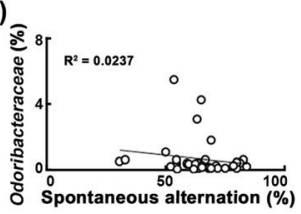

(D)

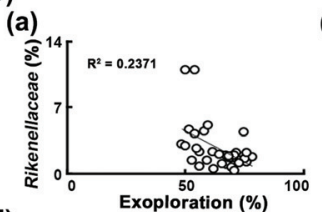

(E)

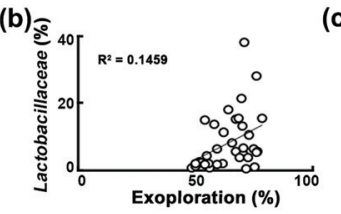

(b)

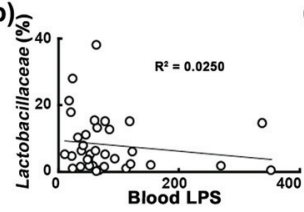

(F)
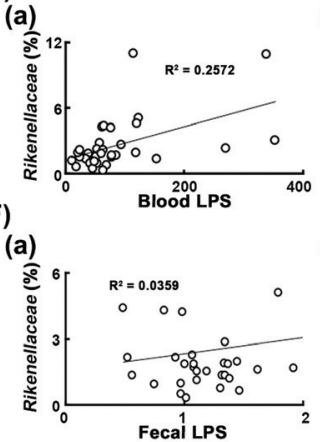

(b)

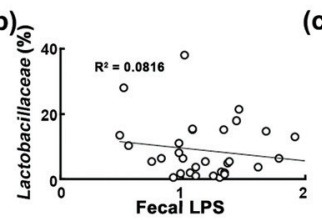

(c)

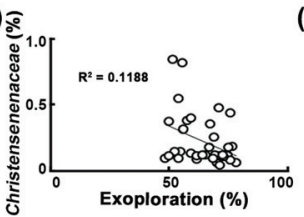

(c)

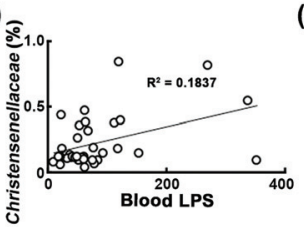

(c)

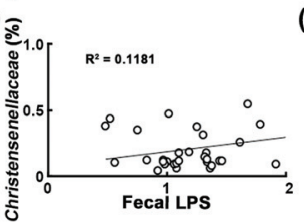

(d)

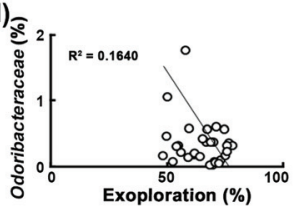

(d)

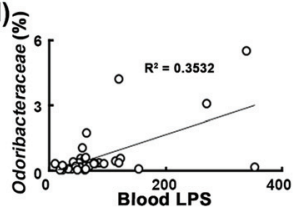

(d)

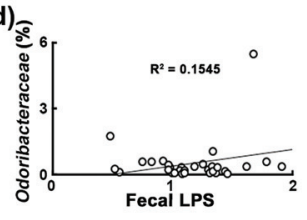

Fig. 5 Effects of NK151 and NK173 on the fecal microbiota composition in mice with Escherichia coli K1-induced cognitive impairment. (A) Effects on fecal microbiota composition: the (a) OTU richness, (b) principal coordinate analysis (PCOA) plot based on Bray-Curtis analysis, and (c) phylum level. (B) The relationship between the spontaneous alternation in the Y-maze task and the PC1 of the gut bacteria PCoA plot. (C) The relationship between the spontaneous alternation in the Y-maze task and gut bacteria. [(a) Rikenellaceae, (b) Lactobacillaceae, (c) Christensenellaceae, and (d) Odoribacteraceae]. (D) The relationship between the blood LPS level and gut bacteria [(a) Rikenellaceae, (b) Lactobacillaceae, (c) Christensenellaceae, and (d) Odoribacteraceae]. (E) The relationship between the blood LPS level and gut bacteria. (F) The relationship between the fecal LPS level and gut bacteria. NK151, NK173, their mixture (NKm), or Escherichia coli K1 (EC, $1 \times 10^{9}$ CFU per mice per day) was orally gavaged. The control group (NC) was treated with the vehicle instead of test agents. Data values were described as mean \pm SD $(n=7)$. ${ }^{\#} p<0.05$ vs. NC. ${ }^{*} p<$ 0.05 vs. EC. 
populations at the genus level; and the Lactobacillus reuteri group population at the species level.

Next, we analyzed the correlation between gut microbiota populations and cognitive function or LPS level in the blood and feces (Fig. 4B-F and ESI Fig. S1, S2 †). The gut microbiota composition (the PC1 of the PCoA plot) was positively correlated with cognitive function in the Y-maze task $\left(R^{2}=0.1723\right.$, $p=0.013)$. At the family level, Lactobacillaceae $\left(R^{2}=0.0256, p=\right.$ 0.359 in the Y-maze task; $R^{2}=0.1459, p=0.024$ in NOR task) and PAC001057_f (Mollicutes; $R^{2}=0.0811, p=0.097$ in Y-maze task; $R^{2}=0.0209, p=0.407$ in the NOR task) populations were positively correlated with cognitive function, while Helicobacteraceae $\left(R^{2}=0.0479, p=0.207\right.$ in the Y-maze task; $R^{2}=0.2585, p=0.002$ in the NOR task), Rikenellaceae $\left(R^{2}=\right.$ $0.2749, p=0.001$ in Y-maze task; $R^{2}=0.2372, p=0.003$ in the NOR task), Ruminococcaceae $\left(R^{2}=0.0160, p=0.469\right.$ in Y-maze task; $R^{2}=0.1149, p=0.047$ in NOR task), Christensenellaceae $\left(R^{2}=0.0077, p=0.618\right.$ in Y-maze task; $R^{2}=0.1188, p=0.043$ in NOR task), and Odoribacteraceae $\left(R^{2}=0.0237, p=0.379\right.$ in the Y-maze task; $R=0.1640, p=0.016$ in the NOR task) populations were negatively correlated with it. At the genus level, Alistipes, Oscillibacter, LT706945_g, and Rikenella populations were negatively correlated with cognitive function, while the Lactobacillus, PAC001118_g, and Roseburia populations were positively correlated with it (Fig. 4C and D). The blood LPS levels were positively correlated with the Christensenellaceae $\left(R^{2}=0.1837, p=0.010\right)$, Odoribacteraceae $\left(R^{2}=0.3532, p \leq\right.$ $0.001)$, and Rikenellaceae $\left(R^{2}=0.2572, p=0.002\right)$ populations, while it was negatively correlated with the Lactobacillaceae population $\left(R^{2}=0.0250, p=0.365\right)$ (Fig. $\left.4 \mathrm{E}\right)$. The fecal LPS levels were positively correlated with the Christensenellaceae $(R=0.1181, p=0.043)$, Odoribacteraceae $\left(R^{2}=0.1545, p=\right.$ $0.020)$, and Rikenellaceae $\left(R^{2}=0.0359, p=0.275\right)$ populations, while it was negatively correlated with the Lactobacillaceae $\left(R^{2}=0.0816, p=0.096\right)$ population (Fig. $\left.4 \mathrm{~F}\right)$.

\subsection{NK151 and NK173 alleviated LPS-induced memory impairment in mice}

The gut microbiota communicates with the immune and neuronal systems through the stimulation of their byproducts such as neurotransmitters and endotoxins. ${ }^{8-10}$ Excessive exposure to the bacterial LPS causes cognitive impairment. ${ }^{10,11}$ Therefore, we examined the effects of NK151 and/or NK173 at a dose of $1 \times 10^{9} \mathrm{CFU}$ per mouse on LPS-induced cognitive impairment in mice (Fig. 6). Intraperitoneal injection of LPS significantly reduced the spontaneous alternation in the Y-maze task and exploration in the NOR task to $74.8 \%$ and $61.3 \%$ of the control group, respectively. However, oral administration of NK151, NK173, or NKm significantly alleviated the LPS-suppressed spontaneous alternation to $94.2 \%, 92.1 \%$ and $97.2 \%$ of the control group, respectively, and exploration to $80.6 \%, 86.3 \%$, and $89.5 \%$, respectively (Fig. $6 \mathrm{~b}$ and c). Oral administration of NK151 and/or NK173 reduced LPS-induced NF-KB activation and IL-1 $\beta$ and TNF- $\alpha$ expression in the hippocampus, while BDNF and IL-10 expression and BDNF to proBDNF expression ratio increased (Fig. 6d-g). They also reduced LPS-induced NF-KB ${ }^{+} / \mathrm{Iba}^{+}$and $\mathrm{LPS}^{+} / \mathrm{Iba}^{+}$cell populations, while the $\mathrm{K} 1$-suppressed $\mathrm{BDNF}^{+} / \mathrm{NeuN}^{+}$cell population increased (Fig. 6h). Furthermore, they significantly decreased LPS and apoE levels in the blood (Fig. 6i and j).

Intraperitoneal injection of LPS also caused colitis: it caused colon shortening, induced myeloperoxidase activity, NF- $\mathrm{KB}$ activation, and TNF- $\alpha$ and IL-1 $\beta$ expression, and decreased IL-10 expression in the colon (Fig. 7). However, oral administration of NK151 and/or NK173 significantly suppressed LPS-induced colon shortening, myeloperoxidase activity, NF- $\mathrm{\kappa B}$ activation, $\mathrm{NF}-\mathrm{\kappa B}^{+} / \mathrm{CD} 11 \mathrm{c}^{+}$cell population, and TNF- $\alpha$ and IL-1 $\beta$ expression, while LPS-suppressed IL-10 expression increased.

\subsection{NK151 and NK173 modulated the gut microbiota composition in mice with LPS-induced cognitive impairment}

We examined whether the attenuation of LPS-induced cognitive impairment by oral administration of NK151 and/or NK173 was associated with the gut microbiota composition. The intraperitoneal injection of LPS shifted the $\beta$-diversity (principal coordinate analysis [PCoA]) while the $\alpha$-diversity was not affected (Fig. 8A and ESI Tables S6-S9†). However, treatment with NK151, NK173, and NKm partially shifted the gut microbiota $\beta$-diversity of LPS-treated mice to those of normal control mice, while the $\alpha$-diversity (OTUs) weakly decreased. Furthermore, LPS treatment increased Firmicutes and Deferribacteres populations, while the Bacteroidetes population decreased. However, oral gavage of NK151, NK173, or NKm reduced Firmicutes, Deferribacteres, and Proteobacteria populations and increased Bacteroidetes population at the phylum level. Furthermore, they increased Muribaculaceae, Bacteroidaceae and Lactobacillaceae, Rikenellaceae, and Odoribacteraceae populations at the family level; PAC000198_g, Bacteroides, Lactobacillus, and PAC002400_g populations at the genus level; and the Lactobacillus murinus group and Bacteroides acidifaciens group populations at the species level. However, they decreased Lachnospiraceae, Prevotellaceae, and Helicobacteraceae populations at the family level, Prevotellaceae_uc, Helicobacter, LLKB_g, and PAC001092_g populations at the genus level, and Helicobacter rodentium group and PAC001072_s populations at the species level.

Next, we analyzed the correlation between the gut microbiota composition and LPS level in the blood or cognitive function (Fig. 8B-E and ESI Fig. S3, S4†). The gut microbiota composition (the PC3 of PCoA plot) was negatively correlated with cognitive function in the Y-maze task function $\left(R^{2}=2836\right.$, 0.001). At the family level, Bacteroidaceae $\left(R^{2}=0.1493, p=\right.$ 0.024 in the Y-maze task; $R^{2}=0.1113, p=0.053$ in the NOR task), Odoribacteraceae $(R=0.0937, p=0.079$ in the Y-maze task; $R^{2}=0.0598, p=0.164$ in the NOR task), and Rikenellaceae $\left(R=0.0176, p=0.455\right.$ in the Y-maze task; $R^{2}=$ $0.1813, p=0.012$ in the NOR task) populations showed a positive correlation with the cognitive function, while Deferribacteraceae $\left(R^{2}=0.0447, p=0.232\right.$ in the Y-maze task; $R=0.2267, p=0.004$ in the NOR task) population was negatively 
(a)

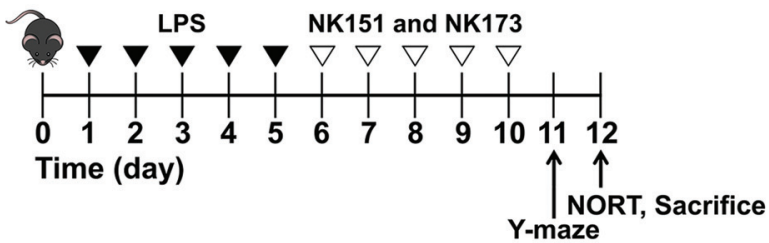

(b)

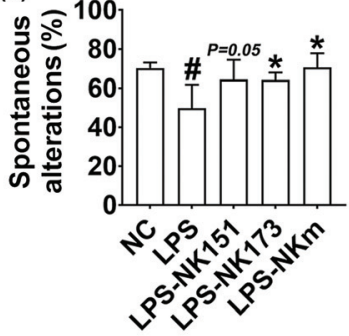

(c)

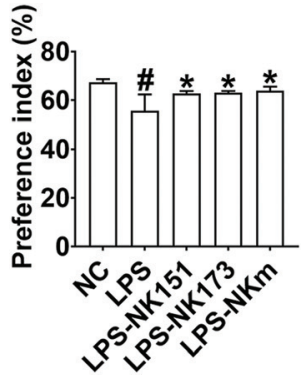

(g)

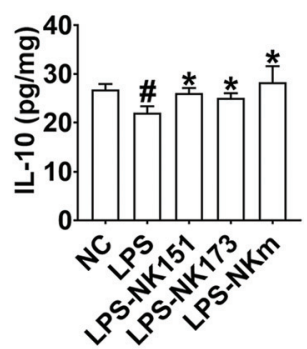

(h)

(e)
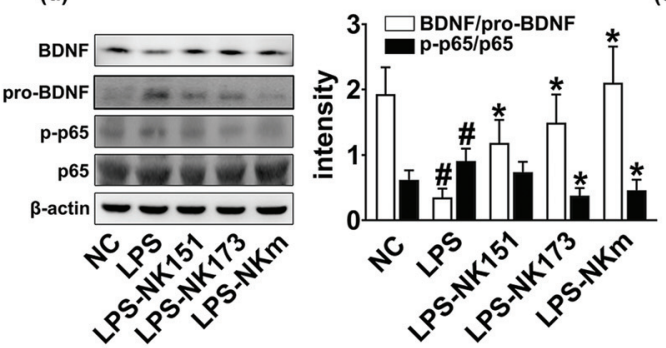

(f)

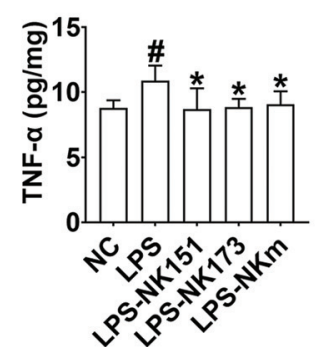

(i)

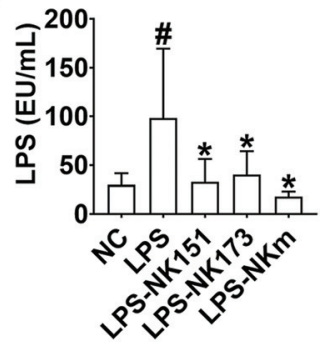

(j)

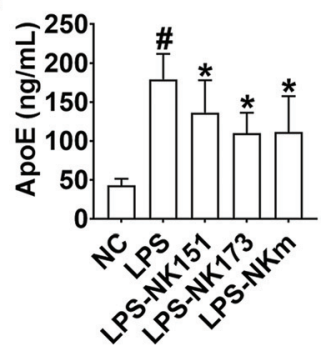

Fig. 6 NK151 and NK173 alleviated LPS-induced cognitive impairment in mice. (a) Experimental procedure. (b) Effects on the spontaneous alternation in the Y-maze task. (c) Effects on the exploration (preference index) in the novel object recognition task. Effects on the BDNF, pro-BDNF, NF- $\mathrm{BB}$ activation, and their intensities (d), IL-1 $\beta$ (e), TNF- $\alpha(f)$, and IL-10 expression (g) and BDNF $/ \mathrm{NeuN}^{+}, \mathrm{LPS}^{+} / \mathrm{lba} 1^{+}$, and NF- $\mathrm{KB}^{+} / \mathrm{lba} 1^{+}$cell populations (h) in the hippocampus. Effects on the LPS (i) and APOE levels (j) in the blood. Cytokines were assayed using ELISA kits. NK151, NK173, and their mixture $(\mathrm{NKm})\left(1 \times 10^{9} \mathrm{CFU}\right.$ per mice per day) were orally gavaged $24 \mathrm{~h}$ after the final peritoneal injection of LPS (10 $\left.\mathrm{ng} \mathrm{kg}^{-1} \mathrm{day}^{-1}\right)$. The control group (NC) was treated with the vehicle instead of test agents. Data values were described as mean \pm SD $(n=7) .{ }^{\#} p<0.05$ vs. NC. ${ }^{*} p<0.05$ vs. LPS.

correlated with it (Fig. 7B and C). At the genus level, Alistipes, Bacteroides, Odoribacter, and KE159600_g populations were positively correlated with the cognitive function; while PAC000692_g (Lachnospiraceae), PAC001372_g, (Lachnospiraceae), and Sporobacter (Ruminococcaceae) populations were negatively correlated with it. The blood LPS levels were negatively correlated with the Odoribacteraceae $\left(R^{2}=\right.$ 0.1152, $p=0.049)$ and Odoribacter $\left(R^{2}=0.1137, p=0.051\right)$ populations, while it was positively correlated with the
PAC001118_g (Lachnospiraceae; $R^{2}=0.1155, p=0.049$ ), PAC001165_g (Lachnospiraceae; $R^{2}=0.1574, p=0.020$ ) populations (Fig. 7D).

\section{Discussion}

The bacterial LPS is a risk factor for the outbreak of sporadic $\mathrm{AD}^{7}$ In the present study, oral gavage of Escherichia coli $\mathrm{K} 1$ or 
(a)

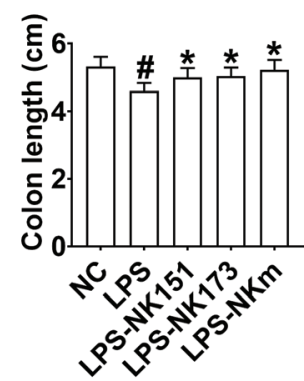

(d)

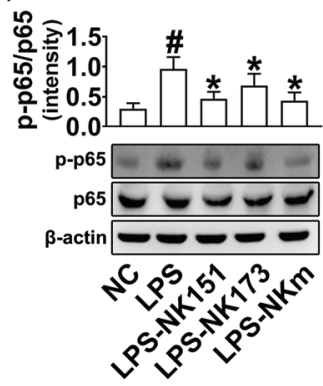

(g)

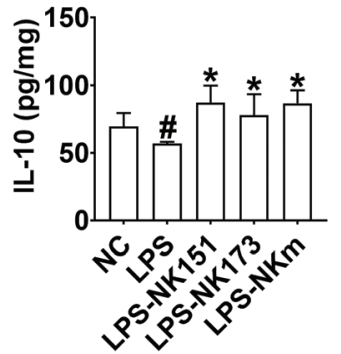

(b)

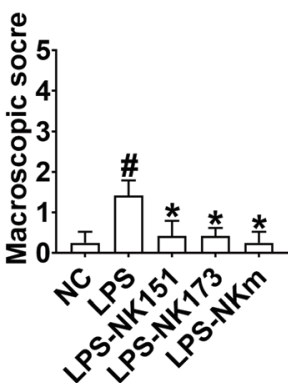

(e)

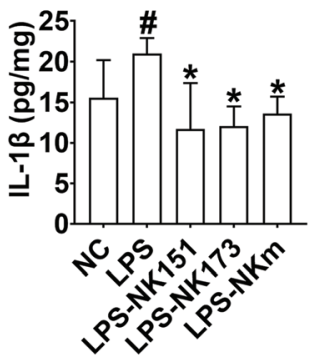

(h)

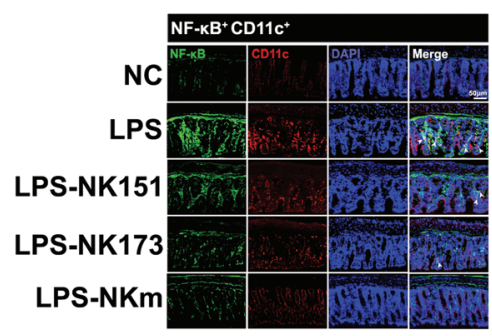

(c)

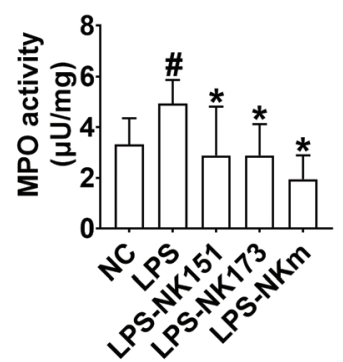

(f)

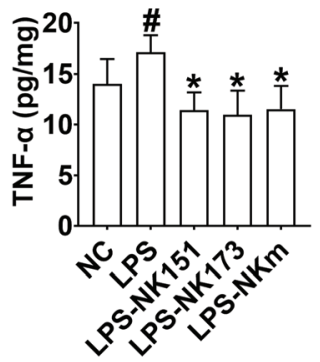

(i)

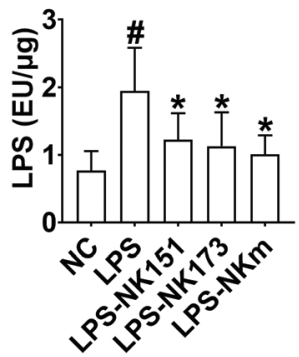

Fig. 7 NK151 and NK173 alleviated colitis in mice with LPS-induced cognitive impairment. (a) Effects on the colon length. (b) Effects on the macro-

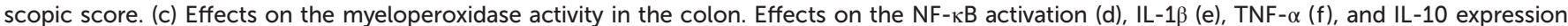
(g) and NF-KB ${ }^{+} / \mathrm{CD} 11 \mathrm{c}^{+}$cell population (h) in the colon. (i) Effects on the LPS in the feces. Cytokines were assayed using ELISA kits. NK151, NK151, NK173, and their mixture $(\mathrm{NKm})\left(1 \times 10^{9} \mathrm{CFU}\right.$ per mice per day) were orally gavaged $24 \mathrm{~h}$ after the final peritoneal injection of LPS (10 $\mathrm{ng} \mathrm{kg}^{-1}$ day $\left.^{-1}\right)$. The control group (NC) was treated with the vehicle instead of test agents. Data values were described as mean \pm SD $(n=7)$. ${ }^{\#} p<0.05$ vs. NC. ${ }^{*} p<$ 0.05 vs. LPS.

intraperitoneal injection of LPS increased the blood LPS level and hippocampal TNF- $\alpha$ and IL- 6 expression and NF- $\mathrm{KB}^{+} / \mathrm{Iba}^{+}$ and $\mathrm{LPS}^{+} / \mathrm{Iba}^{+}$cell populations and decreased the $\mathrm{BDNF}^{+} /$ $\mathrm{NeuN}^{+}$cell population in mice. They also caused cognitive impairment-like behaviors. Bacterial LPS levels are higher in $\mathrm{AD}$ patients than in healthy volunteers. ${ }^{7,28}$ Buttini et al. reported that a peritoneal injection of LPS activated microglia and strongly produced proinflammatory cytokines. ${ }^{29}$ Lee et al. reported that the intraperitoneal injection of LPS caused cognitive impairment and neuroinflammation in mice. ${ }^{24}$ Goel et al. reported that exposure to LPS impaired memory by modulating NF- $\kappa \mathrm{B}-\mathrm{mediated} \mathrm{BDNF}$ expression. ${ }^{30} \mathrm{Kim}$ et al. reported that oral gavage of Escherichia coli $\mathrm{K} 1$ impaired cognitive function in mice and increased the $\mathrm{NF}-\mathrm{KB}^{+} / \mathrm{Iba}^{+}$cell population and TNF- $\alpha$ and IL-1 $\beta$ expression and suppressed the $\mathrm{BDNF}^{+} /$ $\mathrm{NeuN}^{+}$cell population and BDNF expression in the hippocampus. $^{21}$ These suggest that LPS or Escherichia coli $\mathrm{K} 1$ can induce cognitive impairment by suppressing LPS-induced NF- $\kappa \mathrm{B}$ activation-mediated $\mathrm{BDNF}$ expression. Wu et al. reported that chronic exposure to LPS induced AD-like phenotypes in mice. ${ }^{31}$ Lee et al. reported that blood and fecal LPS and hippocampal A $\beta$ expression levels were higher in 5XFAD mice, which exhibited gut dysbiosis, than in healthy control mice. ${ }^{24}$ Zhan et al. reported that LPS induced NF- $\kappa \mathrm{B}$ mediated inflammatory cytokines, which increase $\mathrm{A} \beta$ levels in the $\mathrm{AD}$ brains of rodents. ${ }^{7}$ These results suggest that the overexpression of gut bacterial LPS can induce neuroinflammation and $A \beta$ formation, resulting in the occurrence of $\mathrm{AD}$. We found that LPS or Escherichia coli $\mathrm{K} 1$ also caused colitis: they increased myeloperoxidase activity, TNF- $\alpha$ and IL-6 expression, and NF- $\mathrm{KB}^{+}$/ $\mathrm{Iba}^{+}$cell population in the colon. Furthermore, they caused gut dysbiosis: they increased the Proteobacteria population and LPS level in the gut microbiota. These results support the previous suggestion that the gut microbiota bidirectionally communicates with the brain through the activation of the microbiota-gut-brain axis, linking the cognitive brain system 
(A)

(a)

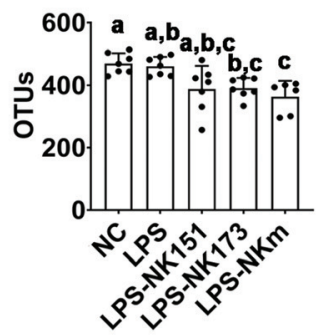

(b)

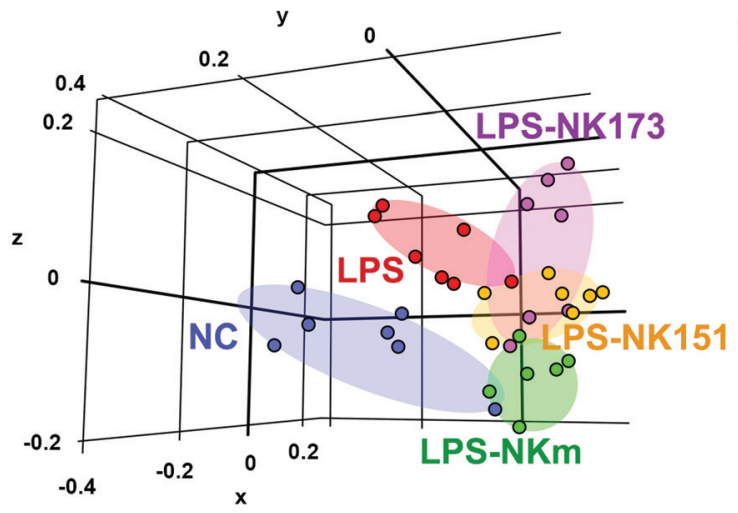

(C)

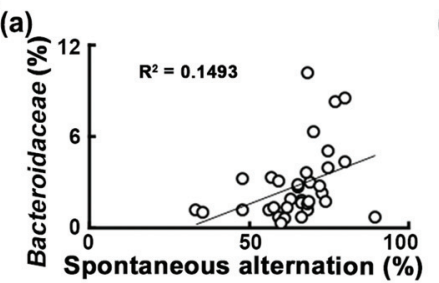

(b)

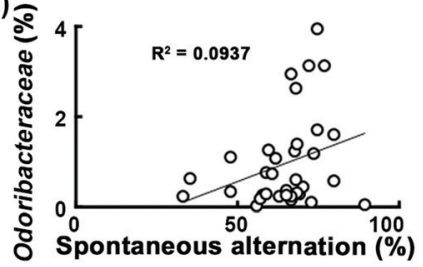

(D) (d)

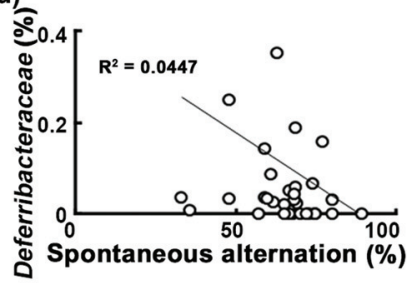

(c)

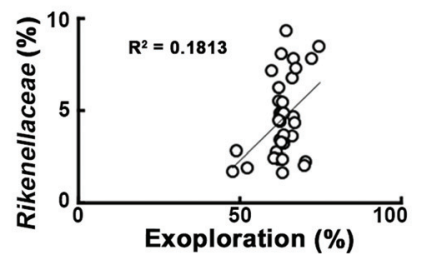

(c)

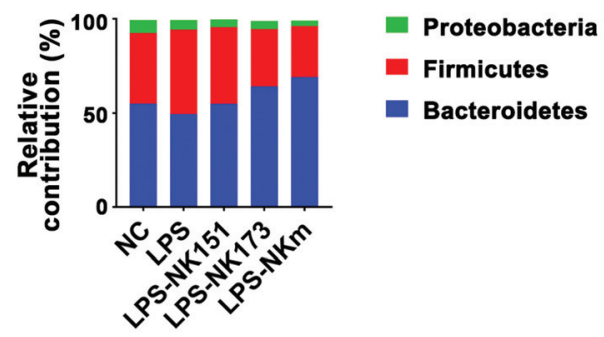

(B) (c)

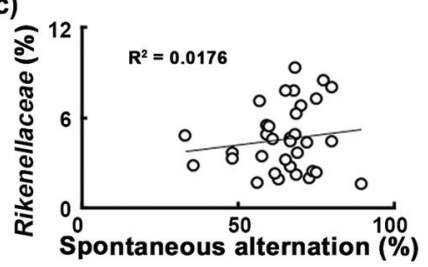

(a)

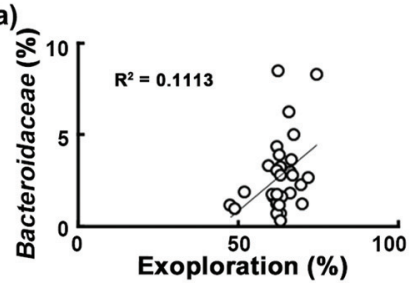

(b)

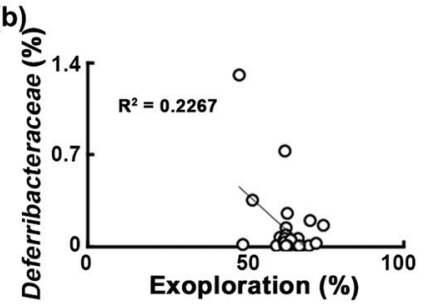

(E)

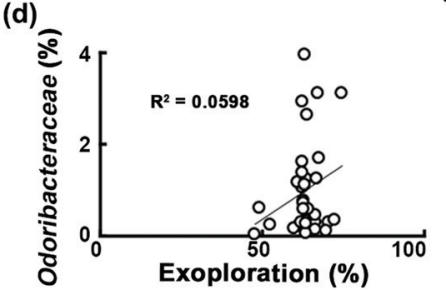

(a)

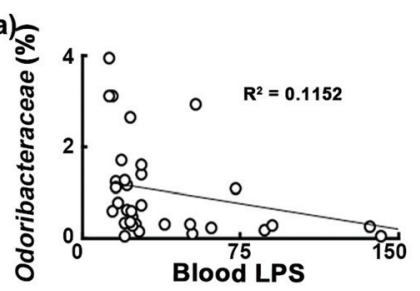

Fig. 8 Effects of NK151 and NK173 on the fecal microbiota composition in mice with LPS-induced cognitive impairment. (A) Effects on fecal microbiota composition: (a) the OTU richness, (b) principal coordinate analysis (PCoA) plot based on Bray-Curtis analysis, and (c) phylum level. (B) The relationship between the spontaneous alternation in the Y-maze task and the PC3 of the gut bacteria PCoA plot. (C) The relationship between the spontaneous alternation in the Y-maze task and gut bacteria [(a) Bacteroidaceae, (b) Odoribacteraceae, (c) Rikenellaceae, and (d) Deferribacteraceae]. (D) The relationship between the exploration in the novel object recognition task and gut bacteria [(a) Bacteroidaceae, (b) Odoribacteraceae, (c) Rikenellaceae, and (d) Deferribacteraceae]. (E) The relationship between the blood LPS level and gut bacteria Odoribacteraceae. NK151, NK173, and their mixture (NKm) $\left(1 \times 10^{9} \mathrm{CFU}\right.$ per mice per day) were orally gavaged $24 \mathrm{~h}$ after the final peritoneal injection of LPS $\left(10 \mathrm{ng} \mathrm{kg}^{-1} \mathrm{day}^{-1}\right)$. The control group (NC) was treated with the vehicle instead of test agents. Data values were described as mean \pm SD $(n=$ 7). ${ }^{\#} p<0.05$ vs. NC. ${ }^{*} p<0.05$ vs. LPS. 
with gut microbiota through the peripheral enteric nervous system. $^{32,33}$

We also found that oral administration of NK151 and/or NK173 suppressed LPS- or Escherichia coli K1-induced NF- $\mathrm{KB}^{+} /$ $\mathrm{Iba}^{+}$and $\mathrm{LPS}^{+} / \mathrm{Iba1}^{+}$cell population and TNF- $\alpha, \mathrm{IL}-1 \beta$, and IL-6 expression in the hippocampus, while the $\mathrm{BDNF}^{+} / \mathrm{NeuN}^{+}$ cell population was increased. Furthermore, they significantly suppressed LPS levels in the blood. TNF- $\alpha$ expression is increased in the blood and cerebrospinal fluid of $\mathrm{AD}$ patients, compared with those in healthy volunteers. ${ }^{34}$ Lew et al. reported that Lactobacillus plantarum P8 reduced cognitive symptoms and the blood TNF- $\alpha$ level in stressed adults. ${ }^{35} \mathrm{Ni}$ et al. reported that Bifidobacterium longum BL986 alleviated learning and memory ability and BDNF expression in aged mice. $^{36}$ A TNF- $\alpha$ blocking agent reduced the risk for Alzheimer's disease (AD) in patients with IBD. ${ }^{37,38}$ These suggest that NK151 and/or NK173 can alleviate LPS- or Escherichia coli K1-induced cognitive impairment by increasing BDNF expression and BDNF to proBDNF expression ratio through the suppression of NF- $\mathrm{KB}$ activation. The combination of NK151 and NK173 synergistically or additively alleviated cognitive impairment. Moreover, although we did not analyse the $A \beta$ expression levels, these probiotics may suppress $A \beta$ expression in the brain by the suppression of LPS-inducible neuroinflammation and $\mathrm{A} \beta$ formation.

Oral gavage of Escherichia coli K1 increased blood LPS and apoE levels in mice. Intraperitoneal injection of LPS also increased the blood apoE levels in mice. Van Oosten et al. reported that the intravenous injection of LPS significantly increased the blood apoE level in mice. ${ }^{39}$ ApoE, which is primarily produced by the liver cells such as Kupffer cells and the brain cells such as astrocytes ${ }^{14,40}$ binds LPS in the blood and most of the LPS is cleared by the liver. ApoE inhibits tolllike receptor (TLR)-3 and TLR-4-mediated inflammatory responses in macrophages. ${ }^{41-43}$ LPS and its producer Escherichia coli $\mathrm{K} 1$ cause systemic inflammation and cognitive impairment in mice. ${ }^{11}$ The concentration of apoE expression in blood is dependent on the expression of LPS-inducible proinflammatory cytokines TNF- $\alpha$ and IL- $1 \beta .{ }^{39}$ These suggest that the translocation of bacterial LPS produced in the gut into the blood can induce the expression of apoE, which degrade LPS in the liver, and, if not excluded, excessive exposure to LPS may cause systemic inflammation and cognitive impairment.

Oral administration of NK151 and/or NK173 NK151 and/or NK173 suppressed the blood apoE level, which is dependent on the fecal and blood LPS levels in mice with LPS- or K1induced cognitive impairment. They also suppressed gut bacterial LPS production in mice and reduced Escherichia coli K1 LPS production in vitro. These suggest that NK151 and/or NK173 may alleviate cognitive impairment by the suppression of systemic inflammation through the suppression of gut bacterial LPS production.

Oral gavage of Escherichia coli $\mathrm{K} 1$ or intraperitoneal injection of LPS caused colitis: it increased myeloperoxidase activity and proinflammatory cytokine expression in the colon.
Moreover, it altered gut microbiota composition: LPS treatment shifted $\beta$-diversity, not $\alpha$-diversity, while Escherichia coli K1 treatment affected $\alpha$ - and $\beta$-diversities. In particular, LPS treatment increased the Firmicutes population, while Escherichia coli K1 treatment suppressed it. However, Escherichia coli $\mathrm{K} 1$ treatment increased Proteobacteria and Bacteroidetes populations. They all increased the LPS level in the blood. Liu et al. reported that the $\gamma$-Proteobacteria population was higher in patients with $\mathrm{AD}$ than in healthy volunteers. ${ }^{44}$ Lee et al. reported that the Proteobacteria population and endotoxin levels were higher in 5XFAD and aged mice than young mice. ${ }^{23}$ These results suggest that cognitive impairment may be dependent on the Proteobacteria population and bacterial LPS levels, which may be a risk factor for the outbreak of cognitive impairment.

NK151 and NK173 significantly alleviated Escherichia coli K1- or LPS-induced gut microbiota alternation and cognitive impairment with colitis. They also suppressed LPS levels in the blood and feces and suppressed the Proteobacteria population in the gut microbiota. They can increase LPS-suppressed Bacteroidaceae, Odoribacteraceae, and Rikenellaceae populations and Escherichia coli K1-suppressed Verrucomicrobiota, Firmicutes, Lactobacillaceae and Bifidobacteriaceae populations. However, they decreased LPSinduced Lachnospiraceae, Prevotellaceae, Helicobacteraceae, and Deferribacteraceae populations and Escherichia coli K1induced Proteobacteria, Rikenellaceae, Christensenellaceae, AC160630_f populations. Thus, the LPS-induced alteration of the gut microbiota composition was different from the Escherichia coli K1-induced one. However, their common features were that stressors LPS and Escherichia coli K1 increased the LPS level in the blood. In mice with Escherichia coli K1induced cognitive impairment, gut bacteria, particularly Lactobacillaceae and PAC001057_f populations were positively correlated with the cognitive function, while Helicobacteraceae, Rikenellaceae, Christensenellaceae, and Odoribacteraceae populations were negatively correlated with it. Of these, Christensenellaceae and Odoribacteraceae were positively correlated with the blood and fecal LPS levels. However, in mice with LPS-induced cognitive impairment, gut bacteria, particularly Bacteroidaceae, Odoribacteraceae, and Rikenellaceae populations showed a positive correlation with the cognitive function, while Deferribacteraceae population was negatively correlated with it. Of these, Odoribacteraceae and Odoribacter were negatively correlated with the blood LPS levels. Overall, the gut microbiota composition was correlated with the cognitive function in mice with Escherichia coli K1-induced cognitive impairment more closely than in mice with LPS-induced cognitive impairment. These results suggest that NK151 and/or NK173 may alleviate cognitive impairment and systemic inflammation by suppressing the blood LPS and through the restoration of the gut microbiota. Moreover, the combination of NK151 and NK173 synergistically or additively alleviated cognitive impairment and systemic inflammation including neuroinflammation and colitis. 


\section{Conclusions}

Oral gavage of NK151, NK173, and their (4:1) mixture (NKm) significantly alleviated Escherichia coli K1- or LPS-induced cognitive impairment-like behaviors and colitis. Treatment with them decreased inflammatory marker expression in the hippocampus and colon, while the $\mathrm{BDNF}^{+} / \mathrm{NeuN}^{+}$cell population increased. They also reduced LPS and apolipoprotein E levels in the blood. Although the exact mechanism needs to be further elucidated, NK151 and/or NK173 can alleviate cognitive impairment with neuroinflammation by upregulating NF- $\kappa \mathrm{B}-$ mediated BDNF expression and BDNF to proBDNF expression ratio through the suppression of fecal and blood bacterial LPS levels, and may thereby be beneficial for therapy of dementia.

\section{Conflicts of interest}

The authors have declared no conflict of interest.

\section{Acknowledgements}

This research was funded by the Medical Research Program through the National Research Foundation of Korea (NRF) funded by the Ministry of Science and ICT (NRF-2017R1A5A2014768) and the Korea Institute of Planning and Evaluation for Technology in Food, Agriculture, Forestry (IPET) through High Value-added Food Technology Development Program funded by Ministry of Agriculture, Food and Rural Affairs (MAFRA 318027-4).

\section{References}

1 M. A. DeTure and D. W. Dickson, The neuropathological diagnosis of Alzheimer's disease, Mol. Neurodegener., 2019, 14,32 .

2 S. A. Gale, D. Acar and K. R. Daffner, Dementia, Am. J. Med., 2018, 131, 1161-1169.

3 M. Dugu, J. Neugroschl, M. Sewell and D. Marin, Review of dementia, Mt. Sinai J. Med., 2003, 70, 45-53.

4 K. Blennow, M. J. de Leon and H. Zetterberg, Alzheimer's disease, Lancet, 2006, 368, 387-403.

5 F. Zhu, C. Li, F. Chu, X. Tian and J. Zhu, Target dysbiosis of gut microbes as a future therapeutic manipulation in Alzheimer's disease, Front. Aging Neurosci., 2020, 12, 544235.

6 K. Kowalski and A. Mulak, Brain-gut-microbiota axis in Alzheimer's disease, J. Neurogastroenterol. Motil., 2019, 25, 48-60.

7 X. Zhan, B. Stamova and F. R. Sharp, Lipopolysaccharide Associates with Amyloid Plaques, Neurons and Oligodendrocytes in Alzheimer's Disease Brain: A Review, Front. Aging Neurosci., 2018, 10, 42.
8 J. F. Cryan, K. J. O’Riordan, K. Sandhu, V. Peterson and T. G. Dinan, The gut microbiome in neurological disorders, Lancet Neurol., 2020, 19, 179-194.

9 J. R. Kelly, G. Clarke, J. F. Cryan and T. G. Dinan, Brain-gutmicrobiota axis: challenges for translation in psychiatry, Ann. Epidemiol., 2016, 26, 366-372.

10 H. M. Jang, H. J. Lee, S. E. Jang, M. J. Han and D. H. Kim, Evidence for interplay among antibacterial-induced gut microbiota disturbance, neuro-inflammation, and anxiety in mice, Mucosal Immunol., 2018, 11, 13861397.

11 H. M. Jang, K. E. Lee, H. J. Lee and D. H. Kim, Immobilization stress-induced Escherichia coli causes anxiety by inducing NF- $\mathrm{BB}$ activation through gut microbiota disturbance, Sci. Rep., 2018, 8, 13897.

12 X. Zhao, F. Cao, Q. Liu, X. Li, G. Xu, G. Liu, Y. Zhang, X. Jang, S. Yi, E. Xu, K. Fan and M. Ma, Behavioral, inflammatory and neurochemical disturbances in LPS and UCMSinduced mouse models of depression, Behav. Brain Res., 2019, 364, 494-502.

13 K. E. Lee, J. K. Kim, S. K. Han, D. Y. Lee, H. J. Lee, S. V. Yim and D. H. Kim, The extracellular vesicle of gut microbial Paenalcaligenes hominis is a risk factor for vagus nervemediated cognitive impairment, Microbiome, 2020, 8, 107.

14 G. C. Brown, The endotoxin hypothesis of neurodegeneration, J. Neuroinflammation, 2019, 16, 180.

15 A. Cattaneo, N. Cattane, V. Begni, C. M. Pariante and M. A. Riva, The human BDNF gene: peripheral gene expression and protein levels as biomarkers for psychiatric disorders, Transl. Psychiatry, 2016, 6, e958.

16 A. E. Autry and L. M. Monteggia, Brain-derived neurotrophic factor and neuropsychiatric disorders, Pharmacol. Rev., 2012, 64, 238-258.

17 T. K. S. Ng, C. S. H. Ho, W. W. S. Tam, E. H. Kua and R. C. M. Ho, Decreased serum brain-derived neurotrophic factor (BDNF) levels in patients with Alzheimer's disease (AD): A systematic review and meta-analysis, Int. J. Mol. Sci., 2019, 20, 257.

18 E. M. Borba, J. A. Duarte, G. Bristot, E. Scotton, A. L. Camozzato and M. L. F. Chavesa, Brain-derived neurotrophic factor serum levels and hippocampal volume in mild cognitive impairment and dementia due to Alzheimer disease, Dement. Geriatr. Cogn. Dis. Extra, 2016, 6, 559-567.

19 Z. Yao, J. M. Mates, A. M. Cheplowitz, L. P. Hammer, A. Maiseyeu, G. S. Phillips, M. D. Wewers, M. V. S. Rajaram, J. M. Robinson, C. L. Anderson and L. P. Ganesan, Bloodborne lipopolysaccharide is rapidly eliminated by liver sinusoidal endothelial cells via high-density lipoprotein, J. Immunol., 2016, 197, 2390-2399.

20 M. Van Oosten, P. C. Rensen, E. S. Van Amersfoort, M. Van Eck, A. M. Van Dam, J. J. Breve, T. Vogel, A. Panet, T. J. Van Berkel and J. Kuiper, Apolipoprotein E protects against bacterial lipopolysaccharide-induced lethality, J. Biol. Chem., 2001, 276, 8820-8824.

21 J. K. Kim, K. E. Lee, S. A. Lee, H. M. Jang and D. H. Kim, Interplay between human gut bacteria Escherichia coli and 
Lactobacillus mucosae in the occurrence of neuropsychiatric disorders in mice, Front. Immunol., 2020, 11, 273.

22 J. J. Jeong, J. Y. Woo, K. A. Kim, M. J. Han and D. H. Kim, Lactobacillus pentosus var. plantarum C29 ameliorates age-dependent memory impairment in Fischer 344 rats, Lett. Appl. Microbiol., 2015, 60, 307-314.

23 H. J. Lee, Y. H. Hwang and D. H. Kim, Lactobacillus plantarum C29-fermented soybean (DW2009) alleviates memory impairment in 5XFAD transgenic mice by regulating microglia activation and gut microbiota composition, Mol. Nutr. Food Res., 2018, 62, e1800359.

24 H. J. Lee, K. E. Lee, J. K. Kim and D. H. Kim, Suppression of gut dysbiosis by Bifidobacterium longum alleviates cognitive decline in 5XFAD transgenic and aged mice, Sci. Rep., 2019, 9, 11814.

25 J. J. Jeong, K. A. Kim, Y. J. Hwang, M. J. Han and D. H. Kim, Anti-inflammaging effects of Lactobacillus brevis OW38 in aged mice, Benefic. Microbes, 2016, 7, 707718.

26 S. W. Yun, J. K. Kim, K. E. Lee, Y. J. Oh, H. J. Choi, M. J. Han and D. H. Kim, A probiotic Lactobacillus gasseri alleviates Escherichia coli-induced cognitive impairment and depression in mice by regulating IL-1beta expression and gut microbiota, Nutrients, 2020, 12, 3441.

27 M. Y. Jeong, H. M. Jang and D. H. Kim, High-fat diet causes psychiatric disorders in mice by increasing Proteobacteria population, Neurosci. Lett., 2019, 698, 51-57.

28 J. M. Hill and W. J. Lukiw, Microbial-generated amyloids and Alzheimer's disease (AD), Front. Aging Neurosci., 2015, 7, 9.

29 M. Buttini, S. Limonta and H. W. Boddeke, Peripheral administration of lipopolysaccharide induces activation of microglial cells in rat brain, Neurochem. Int., 1996, 29, 25-35.

30 R. Goel, S. A. Bhat, K. Hanif, C. Nath and R. Shukla, Angiotensin II receptor blockers attenuate lipopolysaccharide-induced memory impairment by modulation of NF- $\kappa$ Bmediated BDNF/CREB expression and apoptosis in spontaneously hypertensive rats, Mol. Neurobiol., 2018, 55, 1725-1739.

31 Z. Wu, J. Ni, Y. Liu, J. L. Teeling, F. Takayama, A. Collcutt, P. Ibbett and H. Nakanishi, Cathepsin B plays a critical role in inducing Alzheimer's disease-like phenotypes following chronic systemic exposure to lipopolysaccharide from Porphyromonas gingivalis in mice, Brain, Behav., Immun., 2017, 65, 350-361.

32 B. B. Yoo and S. K. Mazmanian, The enteric network: Interactions between the immune and nervous systems of the gut, Immunity, 2017, 46, 910-926.

33 M. Carabotti, A. Scirocco, M. A. Maselli and C. Severia, The gut-brain axis: interactions between enteric microbiota, central and enteric nervous systems, Ann. Gastroenterol., 2015, 28, 203-209.
34 S. L. Montgomery and W. J. Bowers, Tumor necrosis factoralpha and the roles it plays in homeostatic and degenerative processes within the central nervous system, J. Neuroimmune Pharmacol., 2012, 7, 42-59.

35 L. C. Lew, Y. Y. Hor, N. A. A. Yusoff, S. B. Choi, M. S. B. Yusoff, N. S. Roslan, A. Ahmad, J. A. M. Mohammad, M. F. I. L. Abdullah, N. Zakaria, N. Wahid, Z. Sun, L. Y. Kwok, H. Zhang and M. T. Liong, Probiotic Lactobacillus plantarum P8 alleviated stress and anxiety while enhancing memory and cognition in stressed adults: A randomised, double-blind, placebo-controlled study, Clin. Nutr., 2019, 38, 2053-2064.

36 Y. Ni, X. Yang, L. Zheng, Z. Wang, L. Wu, J. Jiang, T. Yang, L. Ma and $\mathrm{Z}$. Fu, Lactobacillus and Bifidobacterium improves physiological function and cognitive ability in aged mice by the regulation of gut microbiota, Mol. Nutr. Food Res., 2019, 63, e1900603.

37 M. Zhou, R. Xu, D. C. Kaelber and M. E. Gurney, Tumor necrosis factor (TNF) blocking agents are associated with lower risk for Alzheimer's disease in patients with rheumatoid arthritis and psoriasis, PLoS One, 2020, 15, e0229819.

38 N. Torres-Acosta, J. H. O’Keefe, E. L. O'Keefe, R. Isaacson and G. Small, Therapeutic potential of TNF-alpha inhibition for Alzheimer's disease prevention, J. Alzheimer's Dis., 2020, 78, 619-626.

39 M. Van Oosten, P. C. N. Rensen, E. S. Van Amersfoort, M. Van Eck, A. M. Van Dam, J. J. Breve, T. Vogel, A. Panet, T. Van Berkel and J. Kuiper, Apolipoprotein E protects against bacterial lipopolysaccharide-induced lethality, J. Biol. Chem., 2001, 276, 8820-8824.

40 K. Patra, A. Giannisis, A. K. Edlund, S. B. Sando, C. Lauridsen, G. Berge, G. R. Grøntvedt, G. Bråthen, L. R. White and H. M. Nielsen, Plasma apolipoprotein E monomer and dimer profile and relevance to Alzheimer's disease, J. Alzheimer's Dis., 2019, 71, 1217-1231.

41 Y. Zhu, A. Kodvawala and D. Y. Hui, Apolipoprotein E inhibits toll-like receptor (TLR)-3- and TLR-4-mediated macrophage activation through distinct mechanisms, Biochem. J., 2010, 428, 47-54.

42 D. Baitsch, H. H. Bock, T. Engel, R. Telgmann, C. MüllerTidow, G. Varga, M. Bot, J. Herz, H. Robenek, A. von Eckardstein and J. R. Nofer, Apolipoprotein E induces antiinflammatory phenotype in macrophages, Arterioscler Thromb Vasc Biol., 2011, 31, 1160-1168.

43 H. Zhang, L. M. Wu and J. Wu, Cross-talk between apolipoprotein E and cytokines, Mediators Inflammation, 2011, 2011, 949072.

44 P. Liu, L. Wu, G. Peng, Y. Han, R. Tang, J. Ge, L. Zhang, L. Jia, S. Yue, K. Zhou, L. Li, B. Luo and B. Wang, Altered microbiomes distinguish Alzheimer's disease from amnestic mild cognitive impairment and health in a Chinese cohort, Brain, Behav., Immun., 2019, 80, 633-643. 